\title{
Field-Enhanced Superconductivity in High-Frequency Niobium Accelerating Cavities
}

\author{
M. Martinello, M. Checchin, A. Romanenko, A. Grassellino, S. Aderhold, \\ S. K. Chandrasekeran, O. Melnychuk, S. Posen, and D. A. Sergatskov \\ Fermi National Accelerator Laboratory, Batavia, Illinois 60510, USA
}

(Received 15 December 2017; published 28 November 2018)

\begin{abstract}
The discovery of nitrogen-doping treatment revealed that the radio frequency surface resistance of niobium resonators may be significantly reduced when nitrogen impurities are dissolved as interstitials in the material. A peculiar behavior exhibited by $\mathrm{N}$-doped cavities is the anti- $Q$ slope, i.e., a reduction in the temperature-dependent component of the surface resistance as a function of the accelerating field. This unusual trend in the surface resistance behavior has been attributed to the presence of interstitial nitrogen in the niobium lattice after the doping treatment. This Letter presents a focused study on the field dependence of the temperature-dependent component of the surface resistance as a function of the cavity resonant frequency. The findings show that the anti- $Q$ slope may appear even in clean niobium cavities if the resonant frequency is high enough, which suggests new routes toward understanding the anti- $Q$ slope effect.
\end{abstract}

DOI: 10.1103/PhysRevLett.121.224801

Introduction.-Superconducting radio frequency (SRF) cavities are a key component of modern particle accelerators to accelerate charged particle beams. The advantage of superconducting technology is its very low dissipation under a radio frequency (rf) field, which allows for continuous operation of accelerating cavities at a high accelerating field $\left(E_{\text {acc }}\right)$. The level of power dissipation in the cavity walls, $P_{\mathrm{d}}$, is inversely proportional to the quality factor, or $Q$ factor $\left(Q_{0}\right)$. This is defined as $Q_{0}=G / R_{s}$, where $G$ is the geometric factor and $R_{s}$ is the surface resistance [1,2].

Record values of $Q$ factors have been achieved with $\mathrm{N}$-doped cavities at $1.3 \mathrm{GHz}$ thanks to the peculiar increase in the $Q$ factor as a function of the field. This is called the anti- $Q$ slope and allows $Q$ factors to be obtained that are up to four times higher than those obtained with previous state-ofthe-art surface treatments at medium field values [3].

The anti- $Q$ slope has been proven to be a consequence of the decrease in the temperature-dependent component of the surface resistance (often referred to as BCS surface resistance) as a function of the accelerating field [3]. This behavior has been attributed to the presence of interstitial nitrogen [3-5]. Two main models have been proposed [6,7] to present a theoretical perspective. However, neither has been experimentally proven, so the anti- $Q$ slope phenomenon is still not fully understood.

In recent years, $R \& D$ on SRF cavities has been focused on $1.3 \mathrm{GHz}$ TESLA-type cavities [8] primarily because of

Published by the American Physical Society under the terms of the Creative Commons Attribution 4.0 International license. Further distribution of this work must maintain attribution to the author(s) and the published article's title, journal citation, and DOI. the interest in realizing many superconducting linear accelerators (LINACs), such as the European Free Electron Laser (EXFEL), Linear Coherent Light Source II (LCLS-II), and International Linear Collider (ILC) [9-11]. However, superconducting accelerating cavities operating at different frequencies are of high interest as well for machines such as the Proton Improvement Plan II (PIP-II), U.S. and the European Spallation Neutron Sources (SNS and ESS), and Facility for Rare Isotope Beams (FRIB) [12-14]. In addition, high-frequency superconducting cavities may be employed in quantum information, astrophysics, and gravitational-wave-detection applications [15-18]. For the reasons mentioned above, the study of the SRF properties of cavities with frequencies other than $1.3 \mathrm{GHz}$ is also of primary interest.

In this Letter, we report the first systematic study on the variation in the microwave surface resistance field dependence as a function of the cavity frequency, in which we analyzed four different resonant frequencies within the range from $650 \mathrm{MHz}$ to $3.9 \mathrm{GHz}$. We verified that the low field value of the temperature-dependent surface resistance varies with the square of the frequency, as predicted by Mattis and Bardeen [19]. In addition, this study revealed that the field dependence of the surface resistance is strongly affected by the frequency and-counterintuitively-that high frequencies promote a decrease in the surface resistance at a medium field. Because of this effect, the decrease in the temperature-dependent surface resistance as a function of the field can also be observed in clean (nondoped) niobium cavities above a certain threshold.

Because of the well-known Mattis and Bardeen frequency dependence of the surface resistance, high-frequency cavities have so far been associated with a low quality factor and thus have not been considered suitable for many applications. Contrary to this misconception, we 
TABLE I. Summary of the frequencies of the studied cavities with the respective surface treatments.

\begin{tabular}{lcccc}
\hline \hline & $650 \mathrm{MHz}$ & $1.3 \mathrm{GHz}$ & $2.6 \mathrm{GHz}$ & $3.9 \mathrm{GHz}$ \\
\hline $\mathrm{BCP}$ & $\mathrm{X}$ & $\mathrm{X}$ & & $\mathrm{X}$ \\
$\mathrm{EP}$ & $\mathrm{X}$ & $\mathrm{X}$ & $\mathrm{X}$ & \\
$120^{\circ} \mathrm{C}$ baking & $\mathrm{X}$ & $\mathrm{X}$ & $\mathrm{X}$ & $\mathrm{X}$ \\
$2 / 6 \mathrm{~N}$ doping & $\mathrm{X}$ & $\mathrm{X}$ & $\mathrm{X}$ & $\mathrm{X}$ \\
\hline \hline
\end{tabular}

showed that unexpectedly high quality factors can be obtained at a medium field with $3.9 \mathrm{GHz} \mathrm{N}$-doped cavities.

Experimental Method.-The study was carried out on elliptical niobium cavities resonating at $650 \mathrm{MHz}$ and 1.3, 2.6, and $3.9 \mathrm{GHz}$. Table I summarizes the surface treatments performed on each cavity. All of the cavities were chemically treated after fabrication, usually via electropolishing (EP), in order to remove about $120-150 \mu \mathrm{m}$ from the inner surface. They were then degassed for about $3 \mathrm{~h}$ at 800 or $900{ }^{\circ} \mathrm{C}$, in a high vacuum furnace. In the case of 3.9 $\mathrm{GHz}$ cavities, buffer chemical polishing (BCP) was performed instead of EP because of the easier applicability to such a small structure. The treatments indicated in Table I were carried out just after the degassing stage. BCP indicates cavities that were buffer chemical polished in order to remove about $40-50 \mu \mathrm{m}$. Analogously, EP indicates that about $40 \mu \mathrm{m}$ are etched from the cavity via electropolishing: $120^{\circ} \mathrm{C}$ baking means that the cavities were fully assembled to be rf tested and were then baked in situ at $120^{\circ} \mathrm{C}$ for about $48 \mathrm{~h}$. $\mathrm{N}$ doping refers to cavities that were treated in the furnace with nitrogen at $800^{\circ} \mathrm{C}$ at a partial pressure of $p=25 \mathrm{~m}$ Torr for $2 \mathrm{~min}$ and then annealed at the same temperature for another $6 \mathrm{~min}$ in a high vacuum before being naturally cooled to room temperature. The doping procedure was always followed by EP removal of $5 \mu \mathrm{m}$.

For each cavity, the surface resistance was decomposed in temperature-dependent $\left(R_{\mathrm{T}}\right)$ and residual resistance $\left(R_{\text {res }}\right)$, and both contributions were calculated as a function of the accelerating field.

Because the temperature-dependent surface resistance exponentially decreases as a function of the temperature, in the case of cavities at low frequencies such as $650 \mathrm{MHz}$ and $1.3 \mathrm{GHz}$, the $R_{\mathrm{T}}$ contribution at $\mathrm{T}=1.5 \mathrm{~K}$ became negligible compared with the residual resistance $R_{\text {res }}$. Therefore, $R_{\mathrm{S}}\left(E_{\mathrm{acc}}, \mathrm{T}=1.5 \mathrm{~K}\right) \simeq R_{\text {res }}\left(E_{\mathrm{acc}}\right)$, and $R_{\mathrm{T}}\left(E_{\mathrm{acc}}, \mathrm{T}=2 \mathrm{~K}\right)$ can be calculated as follows:

$R_{\mathrm{T}}\left(E_{\mathrm{acc}}, \mathrm{T}=2 \mathrm{~K}\right)=R_{\mathrm{S}}\left(E_{\mathrm{acc}}, \mathrm{T}=2 \mathrm{~K}\right)-R_{\mathrm{res}}\left(E_{\mathrm{acc}}\right)$.

For high-frequency cavities, $R_{\mathrm{T}}$ at $\mathrm{T}=1.5 \mathrm{~K}$ cannot be neglected. In this case, $R_{\text {res }}\left(E_{\text {acc }}\right)$ had to be calculated by fitting $R_{\mathrm{S}}\left[\left(E_{\mathrm{acc}}\right), \mathrm{T}\right]$ at different values of accelerating gradients. Because $R_{\text {res }}$ is temperature independent, $R_{\mathrm{S}}(\mathrm{T})$ acquired during the cavity cooldown within a data range between 2 and $1.5 \mathrm{~K}$ can be interpolated by using the following formula:

$$
R_{\mathrm{S}}(\mathrm{T})=\frac{A \omega^{2}}{\mathrm{~T}} e^{-(\Delta / k \mathrm{~T})}+R_{\mathrm{res}},
$$

where $A$ is a material-dependent fitting constant. The $R_{\mathrm{S}}(\mathrm{T})$ curves were acquired at different values of the accelerating field, so the whole curve of the residual resistance can be extrapolated as a function of the accelerating field [20]. Since cavities with slightly different geometries are compared, the accelerating field values were converted in peak surface magnetic field, $B_{\mathrm{pk}}$.

Most of the analyzed cavities were cooled down quickly [21] and in a compensated magnetic field, so the trapped flux contribution was negligible.

The field dependence of the temperature-dependent component of the surface resistance obtained for $1.3 \mathrm{GHz}$ cavities are well in agreement with results published elsewhere $[20,22,23]$. The uncertainty on the estimated value of $R_{\mathrm{T}}$ is usually within $10 \%$ if calculated considering an uncertainty of $4 \%$ on the values of $Q_{0}$ at $2 \mathrm{~K}$ and $10 \%$ on the values of $Q_{0}$ at $1.4 \mathrm{~K}$. This estimation of the uncertainty follows the method described by Melnychuk et al. [24].

Results and Discussion.-Figure 1 shows the temperature-dependent surface resistance measured at $\mathrm{T}=2 \mathrm{~K}$ and $B_{\mathrm{pk}}=20 \mathrm{mT}$ as a function of the resonant frequency $f_{0}$. The experimental points were compared with the theoretical temperature-dependent surface resistance, which was

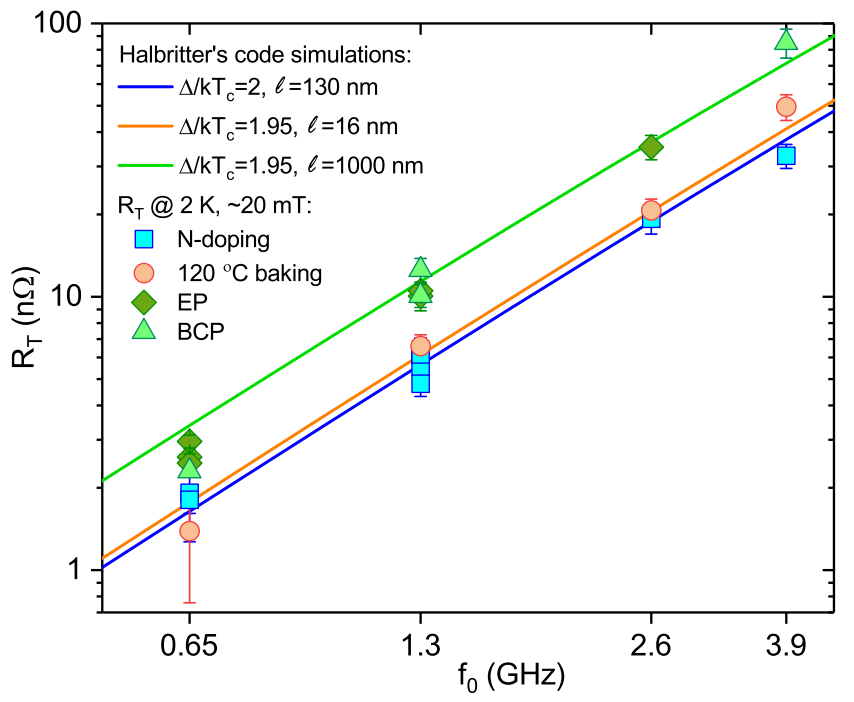

FIG. 1. $R_{\mathrm{T}}$ at $2.0 \mathrm{~K}$ and $5 \mathrm{MV} / \mathrm{m}$ as a function of the resonant frequency. The experimental values for the BCP, EP, N doped, and $120^{\circ} \mathrm{C}$ baked cavities were compared with the Mattis and Bardeen trend of $R_{\mathrm{T}}$ as a function of the resonant frequency simulated with the Halbritter's code [25]. The simulation took into account that each surface treatment modified both the mean free path $\ell$ and reduced energy gap $\Delta / k \mathrm{~T}_{C}$ [4]. 
simulated by using a C++translated version of the Halbritter's code $[25,26]$. Halbritter's numerical calculation of the surface impedance follows the Green's function formalism of Abrikosov, Gor'kov, and Khalatnikov [27]. The Abrikosov-Gor'kov-Khalatnikov theory of surface impedance is based on the BCS theory of superconductivity and lead to the same results as the Mattis-Bardeen theory, developed independently during the same period $[19,28]$. From the Fig. 1 it is possible to observe that, overall, there is a good agreement between the experimental data and the theoretical curves. Only the data of $650 \mathrm{MHz}$ cavities that falls in the clean limit (EP and BCP) are not in agreement with the theoretical curve, within their uncertainty. Deviations from the theoretical trend were also observed in the past in niobium cavities with frequency around $1 \mathrm{GHz}$ [28]; therefore more studies will follow to better understand this phenomenon.

In order to compare the effect of each surface treatment on the temperature-dependent surface resistance field dependence, the data were normalized for the $R_{\mathrm{T}}$ value at a low field (usually around $20 \mathrm{mT}$ ), indicated as $R_{\mathrm{T}}^{0}$. In addition, $R_{\mathrm{T}}$ data of cavities with same resonance frequency and surface treatment were averaged together in order to increase the accuracy of such a comparison.

Figure 2 shows the results of the normalized data $R_{\mathrm{T}} / R_{\mathrm{T}}^{0}$ for the $120^{\circ} \mathrm{C}$ baked cavities. The graph indicates a very similar trend at the low field for cavities with a different frequency. For a peak surface magnetic field greater than approximately $55 \mathrm{mT}$, however, the field dependence starts to deviate depending on the resonant frequency. For example, at $650 \mathrm{MHz}, R_{\mathrm{T}}$ increases very steeply with the field for $B_{\mathrm{pk}}>55 \mathrm{mT}$. The increase is more than that usually observed for $1.3 \mathrm{GHz}$ cavities with the same treatment. On the other hand, the trend is more moderate at $2.6 \mathrm{GHz}$, and at $3.9 \mathrm{GHz}$ the trend is almost reversed with

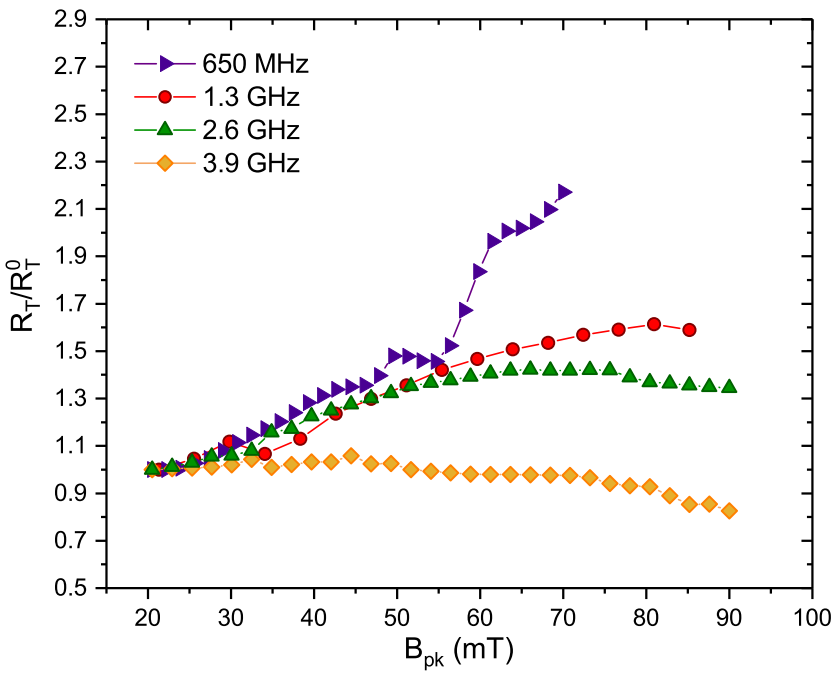

FIG. 2. Normalized data of $R_{\mathrm{T}} / R_{\mathrm{T}}^{0}$ as a function of the peak surface magnetic field for $120^{\circ} \mathrm{C}$ baked cavities at $2.0 \mathrm{~K}$. a slight decrease in $R_{\mathrm{T}}$ with the field. These findings suggest that the field dependence of the temperaturedependent surface resistance is strongly affected by the frequency: higher frequencies yield a smaller $R_{\mathrm{T}}$ contribution at a medium field.

Analogously, Fig. 3 shows the normalized data $R_{\mathrm{T}} / R_{\mathrm{T}}^{0}$ for BCP and EP cavities. In the upper graph the comparison is between $650 \mathrm{MHz}, 1.3$, and $3.9 \mathrm{GHz} \mathrm{BCP}$ cavities, while the lower graph shows results from $650 \mathrm{MHz}, 1.3$, and $2.6 \mathrm{GHz}$ EP cavities. In both cases the results underline that the frequency plays a major role in the $R_{\mathrm{T}}$ field dependence. Also in these cases, for $650 \mathrm{MHz}$ cavities, the temperaturedependent component of the surface resistance increases with the field steeper than for $1.3 \mathrm{GHz}$ cavities. In addition, in contrast to the trend at $1.3 \mathrm{GHz}, R_{\mathrm{T}}$ for $\mathrm{EP}$ and $\mathrm{BCP}$ cavities decreases as a function of the field at 2.6 and 3.9 GHz. These findings represent the first clear proof that the temperature-dependent surface resistance can decrease as a function of the field even in nondoped cavities.

Similar results are observed when $R_{\mathrm{T}} / R_{\mathrm{T}}^{0}$ is compared for $\mathrm{N}$-doped cavities (made with the exact same doping recipe) at $650 \mathrm{MHz}, 1.3 \mathrm{GHz}, 2.6 \mathrm{GHz}$, and $3.9 \mathrm{GHz}$ (Fig. 4). At $650 \mathrm{MHz}$, the temperature-dependent surface resistance of the $\mathrm{N}$-doped cavities slightly increased with

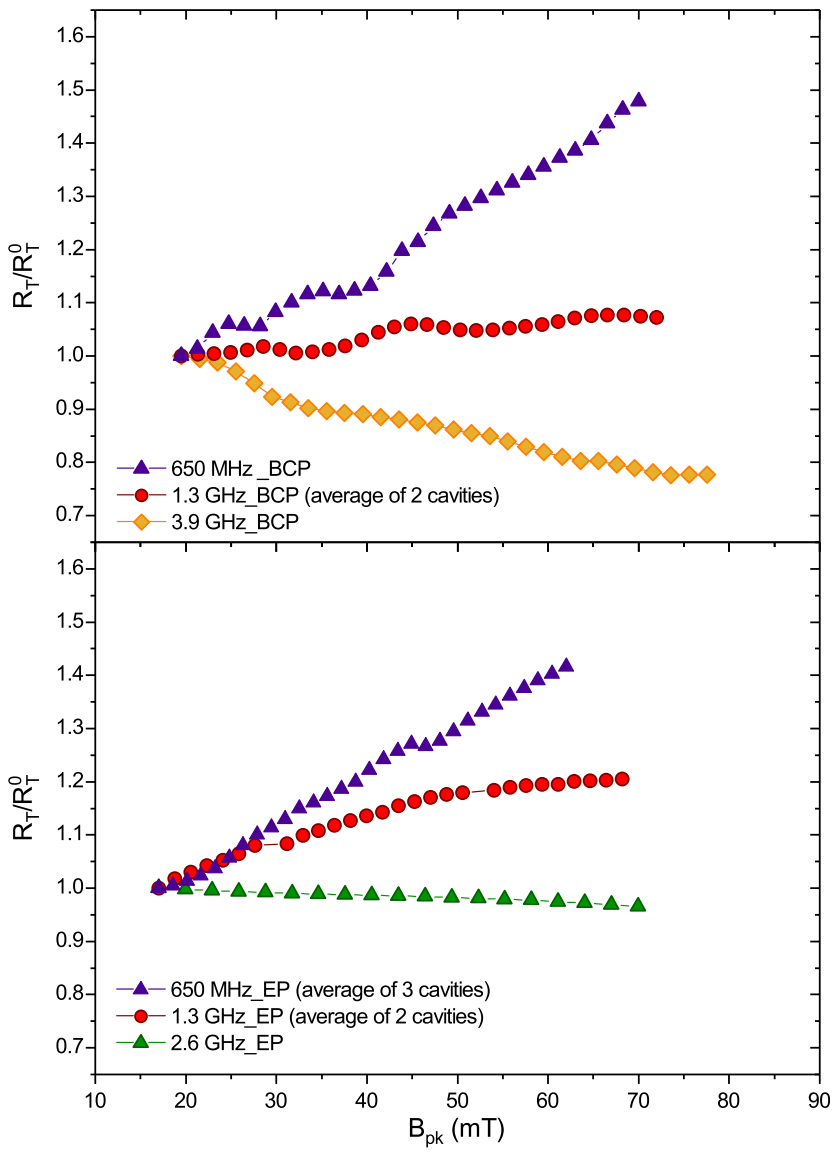

FIG. 3. Normalized data $R_{\mathrm{T}} / R_{\mathrm{T}}^{0}$ as a function of the surface peak magnetic field for BCP and EP cavities at $2.0 \mathrm{~K}$. 


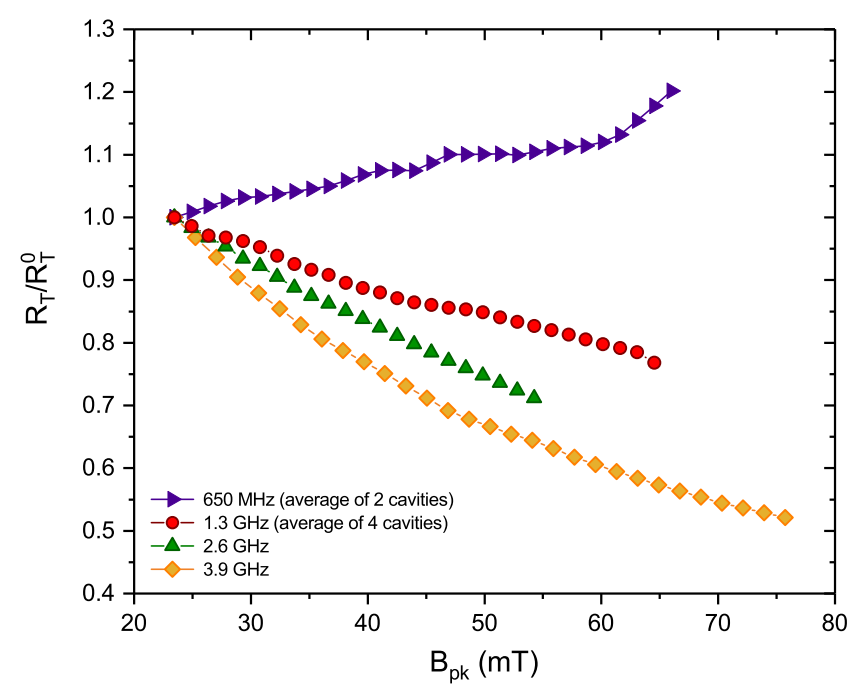

FIG. 4. Normalized data $R_{\mathrm{T}} / R_{\mathrm{T}}^{0}$ as a function of the peak magnetic field for $\mathrm{N}$-doped cavities at $2.0 \mathrm{~K}$.

the field, in contrast with the typical decrease observed at $1.3 \mathrm{GHz}$. On the other hand, at 2.6 and $3.9 \mathrm{GHz}$, the effect of the reversal of $R_{\mathrm{T}}\left(B_{\mathrm{pk}}\right)$ is enhanced. In particular, the temperature-dependent surface resistance substantially decreases for surface peak magnetic field larger than $50 \mathrm{mT}$, at $3.9 \mathrm{GHz}$. Data acquired recently by Koufalis et al. [29] on 1.3 and $2.6 \mathrm{GHz}$ N-doped cavities are well in agreement with our findings.

Thanks to this substantial decrease in the temperaturedependent surface resistance contribution, we observed an unprecedented, high $Q$ factor at a medium field with

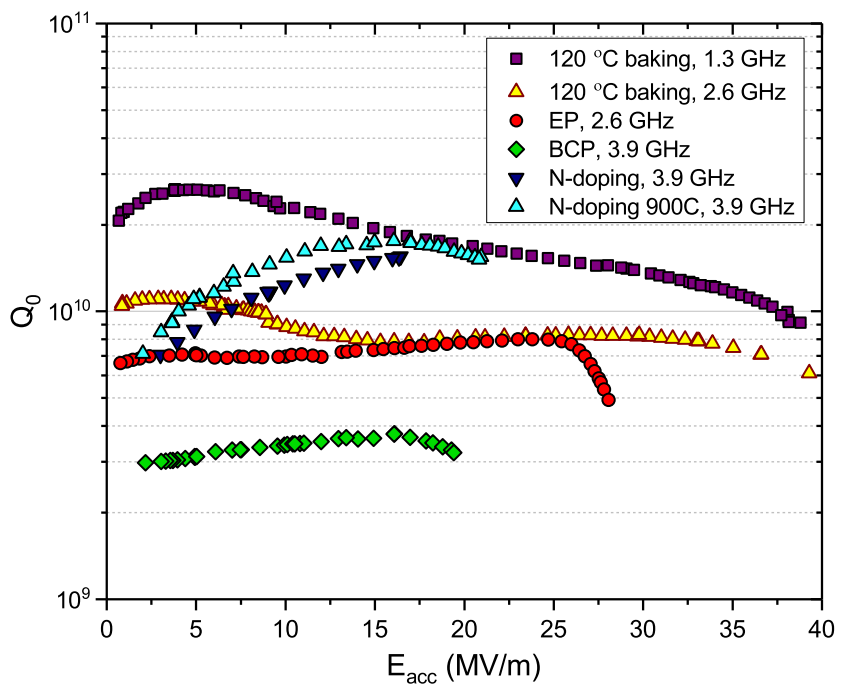

FIG. 5. $Q$ factor versus the accelerating field curves measured for different types of cavities at $2.0 \mathrm{~K}$. $\mathrm{N}$ doping at $900{ }^{\circ} \mathrm{C}$ refers to same procedure of $\mathrm{N}$-doping treatment explained in the experimental method, except that the nitrogen treatment is performed at $900^{\circ} \mathrm{C}$ and is followed by $10 \mu \mathrm{m}$ EP removal.
$\mathrm{N}$-doped $3.9 \mathrm{GHz}$ cavities that reached $Q_{0}>1.5 \times 10^{10}$ at about $17-18 \mathrm{MV} / \mathrm{m}$, as shown in Fig. 5. In the same figure, it is possible to observe also that the curves of the $Q$ factor as a function of the accelerating field, for both the 2.6 GHz EP and the $3.9 \mathrm{GHz} \mathrm{BCP}$ cavity, are characterized by the peculiar anti- $Q$ slope that has previously been observed only in N-doped cavities. A similar result was also observed in a BCP $1.5 \mathrm{GHz}$ cavity made out of medium-purity ingot niobium $(\mathrm{RRR}=125)$ [30].

An important implication of these new findings is that high-frequency cavities may be useful for machines that need to operate with a high accelerating field. For example, we found that the $120^{\circ} \mathrm{C}$ baked $2.6 \mathrm{GHz}$ cavity showed a $Q$ factor approaching that of a $1.3 \mathrm{GHz}$ cavity processed with the same treatment between 35 and $40 \mathrm{MV} / \mathrm{m}$, as shown in Fig. 5.

In summary, we present two major outcomes regarding the decrease in the temperature-dependent surface resistance as a function of the field (origin of the anti- $Q$ slope in $1.3 \mathrm{GHz} \mathrm{N}$-doped cavities): (i) it is more pronounced in high-frequency cavities and suppressed by low-frequency cavities, and (ii) it can be observed even in nondoped cavities if the frequency is high enough.

These findings cannot be easily explained with existing models [6,7]. A recent effort towards extrapolating the frequency dependence from the model proposed by Gurevich [6] by considering the different levels of quasiparticles overheating at different frequencies [31] showed contradictory results compared to our experimental findings.

Based on the experimental data, our hypothesis is that the frequency dependence of the temperature-dependent surface resistance may be a consequence of the quasiparticle distribution being out of equilibrium, with the extent of the nonequilibrium behavior depending on the resonant frequency and on the types and concentration of impurities. The hypothesis that the anti- $Q$ slope of $N$-doped cavities may originate from a deviation of the quasiparticle energy distribution from thermal equilibrium was first proposed by Romanenko [32] and further corroborated in this Letter.

In agreement with the Eliashberg theory [33], superconductors start showing nonequilibrium effects above a certain frequency threshold at which quasiparticles populate high-energy states far from the gap edge. This redistribution of quasiparticles decreases the probability of photon absorption, lowering the dissipation and hence the surface resistance. As previously reported for aluminum cavities [34], such a regime of stimulated superconductivity may manifest itself through a decreasing surface resistance as a function of the field, similarly to what have been observed in niobium SRF cavities.

Conclusions. - This study showed how the temperaturedependent contribution to the surface resistance is a function of the field, resonant frequency, and surface treatment. 
In contrast to expectations based on the study of $1.3 \mathrm{GHz}$ cavities, we showed that $R_{\mathrm{T}}\left(B_{\mathrm{pk}}\right)$ does not always decrease for $\mathrm{N}$-doped cavities and also does not always increase for nondoped cavities. The trend is instead intimately related to both the resonant frequency and type of impurities. Based on these results, high rf frequencies appear to promote a decrease in the temperature-dependent surface resistance at a medium field. This effect allows the observation of the anti- $Q$ slope in $3.9 \mathrm{GHz}$ BCP cavities, and the possibility to reach very high $Q$ factors in $3.9 \mathrm{GHz}$ N-doped cavities. Such unexpected results may promote the employment of high-frequency cavities in future particle accelerators.

In addition, the frequency dependence of the surface resistance suggests that a nonequilibrium distribution of quasiparticles may be responsible for the anti- $Q$ slope in SRF niobium cavities.

This work was supported by the U.S. Department of Energy, Office of High Energy Physics. Fermilab is operated by Fermi Research Alliance, LLC under Contract No. DE-AC02-07CH11359 with the U.S. Department of Energy.

*mmartine@fnal.gov

[1] H. Padamsee, J. Knobloch, and T. Hays, RF Superconductivity for Accelerator (Wiley-VCH Verlag $\mathrm{GmbH}$ and Co., KGaA, Weinheim, 1998).

[2] H. Padamsee, RF Superconductivity: Science, Technology and Applications Vol. II (Wiley-VCH Verlag $\mathrm{GmbH}$ and Co., KGaA, Weinheim, 2009).

[3] A. Grassellino, A. Romanenko, D. Sergatskov, O. Melnychuk, Y. Trenikhina, A. Crawford, A. Rowe, M. Wong, T. Khabiboulline, and F. Barkov, Supercond. Sci. Technol. 26, 102001 (2013).

[4] M. Martinello, A. Grassellino, M. Checchin, A. Romanenko, O. Melnychuk, D. A. Sergatskov, S. Posen, and J. F. Zasadzinski, Appl. Phys. Lett. 109, 062601 (2016).

[5] D. Gonnella, J. Kaufman, and M. Liepe, J. Appl. Phys. 119, 073904 (2016).

[6] A. Gurevich, Phys. Rev. Lett. 113, 087001 (2014).

[7] B. P. Xiao, C. E. Reece, and M. J. Kelley, Physica (Amsterdam) 490C, 26 (2013).

[8] B. Aune et al., Phys. Rev. ST Accel. Beams 3, 092001 (2000).

[9] N. M. Waldrop, Nature (London) 505, 604 (2014).

[10] M. Yabashi and H. Tanaka, Nat. Photonics 11, 12 (2017).

[11] M. Harrison, International Linear Collider Technical Design Report Vol. 1 through 4 (Brookhaven National Laboratory [BNL], Upton, NY, 2013).

[12] M. Ball et al., The PIP-II Conceptual Design Report, edited by V. Lebedev, FERMILAB-DESIGN-2017-01 (2017).
[13] T. E. Mason et al., Physica (Amsterdam) B385-386, 955 (2006).

[14] G. Bollen, AIP Conf. Proc. 1224, 432 (2010).

[15] J. Jaeckel and A. Ringwald, Phys. Lett. B 659, 509 (2008).

[16] H. Paik, D. I. Schuster, L. S. Bishop, G. Kirchmair, G. Catelani, A. P. Sears, B. R. Johnson, M. J. Reagor, L. Frunzio, L. I. Glazman, S. M. Girvin, M. H. Devoret, and R. J. Schoelkopf, Phys. Rev. Lett. 107, 240501 (2011).

[17] M. Reagor, W. Pfaff, C. Axline, R. W. Heeres, N. Ofek, K. Sliwa, E. Holland, C. Wang, J. Blumoff, K. Chou, M. J. Hatridge, L. Frunzio, M. H. Devoret, L. Jiang, and R. J. Schoelkopf, Phys. Rev. B 94, 014506 (2016).

[18] A. Romanenko and D. I. Schuster, Phys. Rev. Lett. 119, 264801 (2017).

[19] D. C. Mattis and J. Bardeen, Phys. Rev. 111, 412 (1958).

[20] A. Romanenko and A. Grassellino, Appl. Phys. Lett. 102, 252603 (2013).

[21] A. Romanenko, A. Grassellino, O. Melnychuk, and D. A. Sergatskov, J. Appl. Phys. 115, 184903 (2014).

[22] D. Gonnella, S. Aderhold, A. Burrill, E. Daly, K. Davis, A. Grassellino, C. Grimm, T. Khabiboulline, F. Marhauser, O. Melnychuk, A. Palczewski, S. Posen, M. Ross, D. Sergatskov, A. Sukhanov, Y. Trenikhina, and K. M. Wilson, Nucl. Instrum. Methods Phys. Res., Sect. A 883, 143 (2018).

[23] T. Okada, in TESLA Technology Collaboration meeting, RIKEN Nishina Center, Japan, 2018, https://indico.desy.de/ indico/event/20010/session/35/contribution/67.

[24] O. Melnychuk, A. Grassellino, and A. Romanenko, Rev. Sci. Instrum. 85, 124705 (2014).

[25] J. Halbritter, Institut für Experimentelle Kernphysik (IEKP), Report No. KFK-Extern 03/70-06, 1970.

[26] J. Halbritter, Z. Phys. 266, 209 (1974).

[27] A. Abrikosov, L. Gor'kov, and I. Khalatnikov, ZhETF 35, 265 (1958)[Sov. Phys. JETP 8, 182 (1959)].

[28] J. P. Turneaure, J. Halbritter, and H. A. Schwettman, J. Supercond. 4, 341 (1991).

[29] P. Koufalis, M. Liepe, J. Maniscalco, and T. Oseroff, in 9th International Particle Accelerator Conference, Vancouver, BC, Canada, 2018 (JACoW Publishing, Geneva, 2018) p. 2451.

[30] G. Ciovati, P. Dhakal, and G. R. Myneni, Supercond. Sci. Technol. 29, 064002 (2016).

[31] J. T. Maniscalco, F. Furuta, D. L. Hall, P. N. Koufalis, and M. Liepe, in 8th International Particle Accelerator Conference, Copenhagen, Denmark, 2017 (JACoW Publishing, Geneva, 2017) p. 3609.

[32] A. Romanenko, in 27th International Linear Accelerator Conference, Geneva, Switzerland, 2014, http://accelconf .web.cern.ch/AccelConf/linac2014/talks/tuioc02_talk.pdf.

[33] G. M. Eliashberg, ZhETF Pis. Red. 11, 186 (1970) [JETP Lett. 11, 114 (1970).]

[34] P. J. De Visser, D. J. Goldie, P. Diener, S. Withington, J. J. A. Baselmans, and T. M. Klapwijk, Phys. Rev. Lett. 112, 047004 (2014). 